\title{
Mathematical simulation of operation of multi- chamber arrester for lightning protection of power lines: calculation of thermophysical properties of nonequilibrium plasma
}

\author{
Dmitriy Ivanov ${ }^{1, *}$, Vladimir Skornyakov ${ }^{1}$, Irina Savelieva ${ }^{1}$, Mikhail Korotkikh $^{1}$, Vyacheslav \\ Shestakov ${ }^{1}$, Dirk Uhrlandt ${ }^{2}$, and Georgy Podporkin ${ }^{3}$ \\ ${ }^{1}$ Peter the Great St. Petersburg Polytechnic University, 195251 St. Petersburg, Russia \\ ${ }^{2}$ INP Greifswald, 17489, Greifswald, Germany \\ ${ }^{3}$ Streamer International AG, 191024, St. Petersburg, Russia
}

\begin{abstract}
Protection of important energy facilities from direct lightning impact requires the creation of special devices for lightning protection in particular multi-chamber arresters. To improve the efficiency of multichamber arrester operation it is necessary to perform mathematical simulation of the physical processes that take place inside such devices. To develop a mathematical model of arc discharge it is necessary to know the thermodynamic and transport properties of the plasma depending on temperature and pressure. In the article the dependences of the thermodynamic and transport properties of plasma in a multi-chamber arrester were obtained for the two-temperature plasma model for the electron temperature range $T_{e}=300-30000 \mathrm{~K}$, the nonequilibrium degree range is $1-5$, and the pressure range $p=0.3-1$ atm.
\end{abstract}

\section{Introduction}

Protection of important energy facilities from direct lightning impact requires the creation of special devices for lightning protection.

Currently, a new promising way to protect overhead power lines from lightning impact is the use of multi-chamber arresters [1-3]. The multi-chamber arrester consists of a large number of series-connected chambers in which a pulsed arc discharge occurs during breakdown. Such a discharge is accompanied by erosion of the electrodes material and ablation of the material of the discharge chamber. During that process an increased pressure occurs in the chamber leading to the formation of a plasma jet from the discharge chamber and to the extinction of the electric arc [1].

To improve the efficiency of multi-chamber arrester operation it is necessary to perform studies of the physical processes that take place inside such devices. Along with experimental methods [4], theoretical studies using mathematical modeling become widespread [5-10].

* Corresponding author: d.ivanov@ spbstu.ru 
The data obtained during experiments are used in the mathematical model as initial data as well as for the purpose of correcting the model.

To develop a mathematical model of a pulsed arc discharge it is necessary to know the thermodynamic and transport properties of the plasma depending on temperature and pressure. To calculate these properties, in turn, it is necessary to know a plasma composition i.e. dependences of number densities of plasma components on temperature (and pressure). The calculation of the composition and properties of such plasma for a model that assumes local thermodynamic equilibrium was carried out earlier [5].

Currently, there is the problem of application of arresters for power lines located in mountainous areas where in some cases the pressure may be 0.3-0.8 of the atmospheric pressure. The extinction of an electric arc in the arresters under such conditions becomes difficult, which is caused by the stable maintenance of the arc under reduced pressure. Therefore, the creation of lightning arresters requires significant adjustments.

The purpose of this article is to bring the conditions of arc existence at a mathematical model into accordance with the actual operating conditions of the object of investigation. To do that the composition and properties of the electric arc under reduced pressure as well as under conditions of disturbance of local thermodynamic equilibrium will be calculated.

\section{Methods}

The main assumptions for calculation of the thermophysical properties of plasma using a two-temperature model can be found in [11].

\subsection{Calculation of plasma composition}

The method of calculation of the composition of thermal (equilibrium) plasma is described in sufficient details in the literature [12-14].

In the case of the two-temperature approximation the plasma composition depends on the electron temperature $T_{e}$, the temperature $T_{h}$ of the heavy species and the pressure $p$. In practice, the dependence of plasma composition on temperature $T_{e}$ and the parameter $\theta=T_{e} / T_{h}$ is usually calculated at constant pressure.

The method of formation a system of equations describing composition in a chemically reacting medium is usually founded on two different approaches, one of which is based on the equations of mass action law using equilibrium constants [13, 14], and the other one - on finding the minimum of the thermodynamic potential of the system [11, 13, 15-17]. Further only the first method will be described.

If the number of individual components taken into account in the calculation (including electrons $e$ ) is equal $m$, and the number of chemical elements forming the system is equal $n$, then to determine the composition, one can compose $(m-n-1)$ equations of the mass action law, i.e. write expressions for the equilibrium constants. For neutral components these equations most often describe the dissociation of molecules into atoms:

$$
K_{j}(T, p)=\prod_{i=1}^{n} \frac{p_{i}^{a_{i, j}}}{p_{j}},
$$

where $p_{i}$ are partial pressures of elements (atoms); $p_{j}$ are partial pressures of complex components (molecules); $a_{i, j}$ is a matrix of stoichiometric coefficients of the reactions of decomposition of complex components into atoms; $K_{j}(T, p)$ is the equilibrium constant of the corresponding reaction of the dissociation of the complex component into atoms.

Here, the following rule is valid for two-temperature plasma [11]: if electrons participate in the reaction (for example, in the case of formation of a molecular ion) then the electron 
temperature $T_{e}$ is used in formula (1) instead of $T: T \equiv T_{e}$; otherwise, the temperature $T_{h}$ of the heavy species is used: $T \equiv T_{h}$.

For atomic ions the mass action law is expressed by the well-known Saha equation which for the two-temperature plasma model has the following form [11]:

$$
\frac{n_{i} \cdot n_{e}}{n_{a}}=\frac{Z_{i}\left(T_{e}\right) \cdot \mathrm{g}_{e}}{Z_{a}\left(T_{e}\right)} \cdot\left(\frac{2 \pi \cdot m_{e} \cdot k \cdot T_{e}}{h^{2}}\right)^{3 / 2} \cdot \exp \left(-\frac{E_{\text {ioniz }}-\Delta E}{k \cdot T_{e}}\right),
$$

where $n_{i}, n_{e}, n_{a}$ are the number densities of ions, electrons, atoms; $Z_{i}\left(T_{e}\right), Z_{a}\left(T_{e}\right)$ are the partition functions of ion and atom; $g_{e}=2$ is the statistical weight of electron; $m_{e}$ is electron mass; $k$ is Boltzmann constant; $h$ is Planck's constant; $E_{\text {ioniz }}$ is ionization energy; $\Delta E$ is reduction of ionization potential.

The partition functions of ions and atoms are determined by the following formula [11]:

$$
Z\left(T_{e}\right)=\sum_{i} \mathrm{~g}_{i} \exp \left(-\frac{E_{i}-E_{0}}{k T_{e}}\right),
$$

where $g_{i}$ is the statistical weight of $i$-th electron level of the ion (atom); $E_{i}$ is the energy of the $i$-th level of the ion (atom); $E_{0}$ is the energy of the ground state of the ion (atom).

The most complete information about electronic states of ions and atoms is contained in the NIST database [18]. It should be noted that in the case of ionization reactions it is preferable to use expression (3) rather than to use tabular data from reference books [19, 20].

The reduction of ionization potential due to the influence of charged particles was estimated in the Debye-Hückel approximation [21].

Dalton's law on the sum of partial pressures should also be included into the system of equations of thermodynamic equilibrium for an ideal gas mixture:

$$
\sum_{j=1}^{m} p_{j}=p
$$

In the case of the two-temperature plasma model, when electrons have a temperature $T_{e}$ (and therefore their partial pressure is $p_{e}=n_{e} k T_{e}$ ), while all other (heavy) species have a temperature $T_{h}$ (and therefore their partial pressure is $p_{j}=n_{j} k T_{h}$ ), Dalton's law is written as follows:

$$
n_{e} k T_{e}+\sum_{j=2}^{m} n_{j} k T_{h}=p
$$

Another equation that is necessary for calculation of the plasma composition (i.e. substance containing charged species such as ions and electrons) is the equation of charge conservation law (quasi-neutrality equation):

$$
\sum_{i} a_{i} \cdot n_{i+}=\sum_{j} a_{j} \cdot n_{j-}
$$

where $a_{i}$ and $n_{i+}$ are the charge and number density of positively charged ions, and $a_{j}$ and $n_{j+}$ are the charge and number density of negatively charged ions (as well as electrons).

To close the system of equations, it is necessary to write $(n-1)$ equations of material balances:

$$
\frac{\sum_{i=1}^{m} a_{g_{i}} n_{i}}{\sum_{i=1}^{m} a_{k_{i}} n_{i}}=\frac{G}{K},
$$

where $a_{g_{i}}$ and $a_{k_{i}}$ are the number of atoms of the kind $g$ and $k$ respectively in the molecule of the $i$-th substance; $G$ and $K$ are the total number of atoms of the kind $g$ and $k$ in the plasma. 
The resulting system of equations of thermodynamic equilibrium of the form (1), (2), (5) - (7) is a system of nonlinear algebraic equations. This system was solved by a modified Newton method for increments of logarithms of unknowns [14].

\subsection{Calculation of thermodynamic and transport properties}

A method of calculation of thermodynamic and transport properties for two-temperature plasma can be found in the references [21-29].

\subsection{Initial data for calculation}

Silicone rubber has the following chemical formula:

$\left(\mathrm{C}_{2} \mathrm{H}_{6} \mathrm{SiO}\right)_{n}$

Using this information and considering possible cases of the electrode material $(\mathrm{Cu}, \mathrm{W}$, Fe) the 85 components were taken into calculation.

The equilibrium constants for the equations of the mass action law were taken from [19], and the partition functions of atoms and ions were calculated based on NIST data [18]. Enthalpies of individual components were taken from [19] to calculate the thermodynamic properties.

To calculate the transport properties, it is necessary to find data on collision cross sections for each pair of species. The book [29] was used as the main source for specifying collisions between two neutral species and between neutral and charged components. If the required cross section was not found in the book [29] then we acted as follows. When considering collisions between two neutral components it was assumed that the cross section can be described by the Lennard-Jones interaction potential [23], the main interaction parameters were taken from [30,31] using rules given in [31]. When considering collisions between neutral and charged components it was assumed that the cross section can be described by the polarization potential, the parameters of which were taken from [30, 31]. The collision of two charged components was described by a screened Coulomb potential [32].

\section{Results and Discussion}

A series of calculations were carried out for a pressure range of $0.3-1.0 \mathrm{~atm}$ and a temperature range of 1000-30 $000 \mathrm{~K}$ with the following proportions of basic chemical elements included into the system:

$$
\mathrm{Si}: \mathrm{O}: \mathrm{C}: \mathrm{H}=1: 1: 2: 6 ; \quad \mathrm{Fe}: \mathrm{O}=1: 10 \text {. }
$$

These ratios were determined using a condition that the walls of the discharge chamber are made of silicone rubber and the electrodes are made of steel.

The ratio $\mathrm{Fe}: \mathrm{O}=1: 10$ was made on the basis of experimental studies of the plasma composition at a discharge in the chamber of the multi-chamber arrester.

The calculation results are presented in Figs. 1-9.

The calculation results show that the dependences of plasma properties on temperature have highly non-linear view which is explained by a complex chemical composition. The nonequilibrium degree (parameter $\theta$ ) also strongly influences the thermophysical properties.

In general, it can be said that the character of the dependences is consistent with similar dependencies known from the literature [21, 25-31]. 
a)

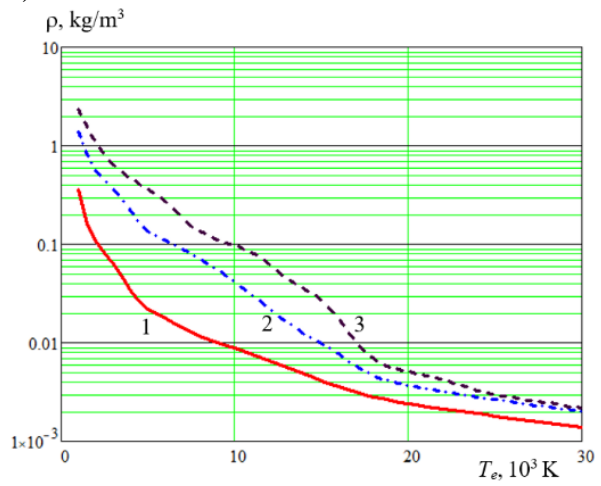

b)

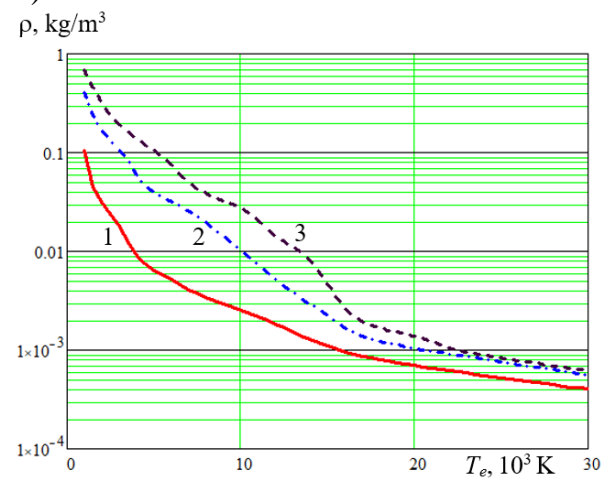

Fig. 1. Mass density dependence on electron temperature at different values of parameter $\theta(1-\theta=1$; $2-\theta=3 ; 3-\theta=5$ ) for different plasma pressures: $\mathrm{a}-1 \mathrm{~atm}, \mathrm{~b}-0.3 \mathrm{~atm}$

a)

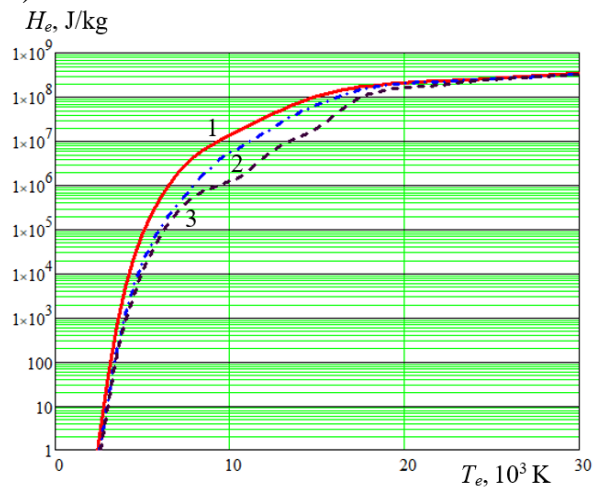

b)

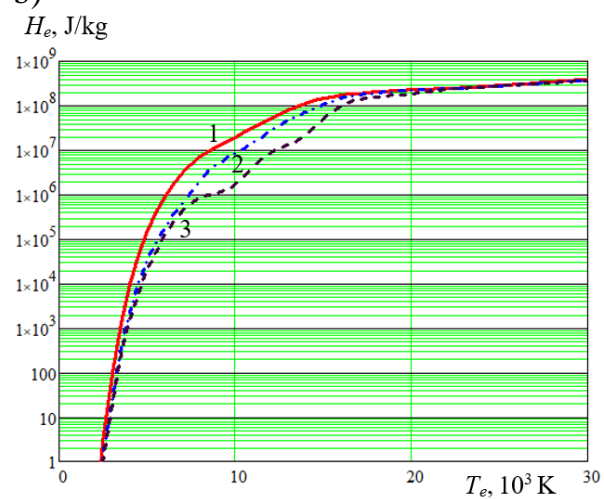

Fig. 2. Electron enthalpy dependence on electron temperature at different values of parameter $\theta$ $(1-\theta=1 ; 2-\theta=3 ; 3-\theta=5)$ for different plasma pressures: $\mathrm{a}-1 \mathrm{~atm}, \mathrm{~b}-0.3 \mathrm{~atm}$

a)

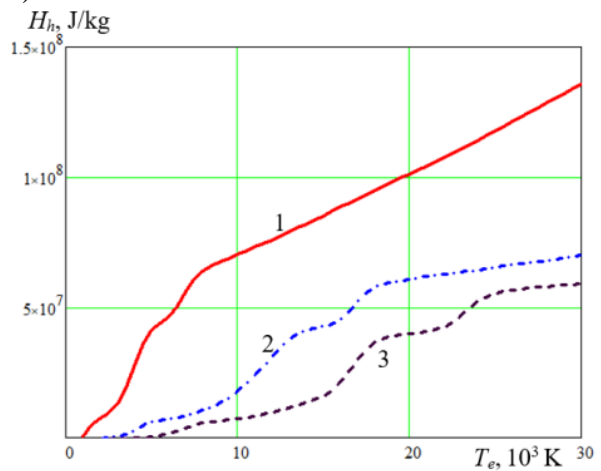

b)

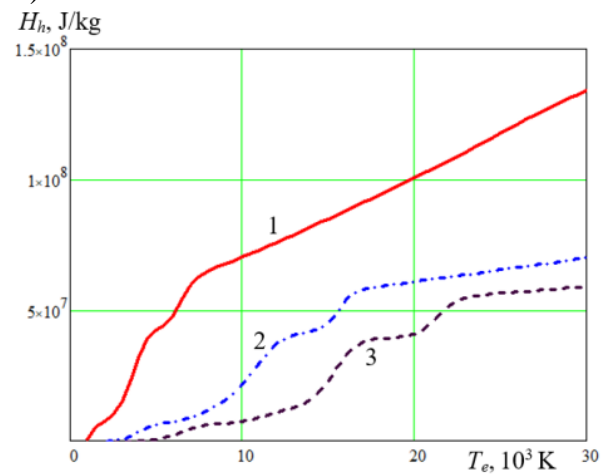

Fig. 3. Heavy particles enthalpy dependence on electron temperature at different values of parameter $\theta(1-\theta=1 ; 2-\theta=3 ; 3-\theta=5)$ for different plasma pressures: $\mathrm{a}-1 \mathrm{~atm}, \mathrm{~b}-0.3 \mathrm{~atm}$ 
a)

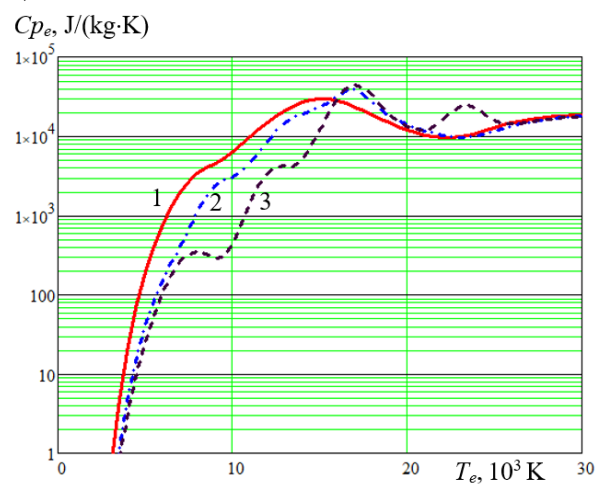

b)

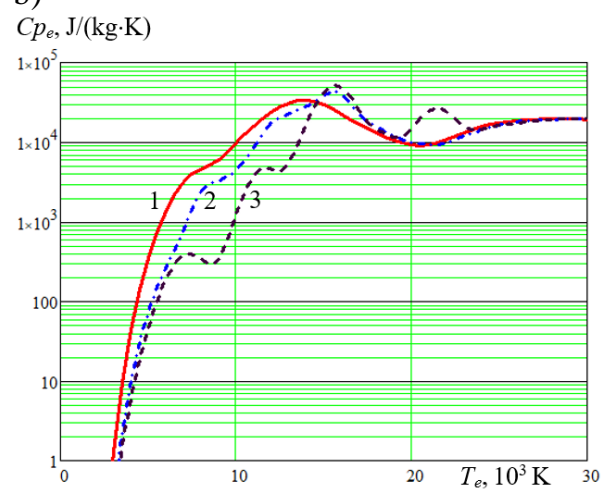

Fig. 4. Electron specific heat dependence on electron temperature at different values of parameter $\theta$ $(1-\theta=1 ; 2-\theta=3 ; 3-\theta=5)$ for different plasma pressures: $a-1 \mathrm{~atm}, \mathrm{~b}-0.3 \mathrm{~atm}$

a)

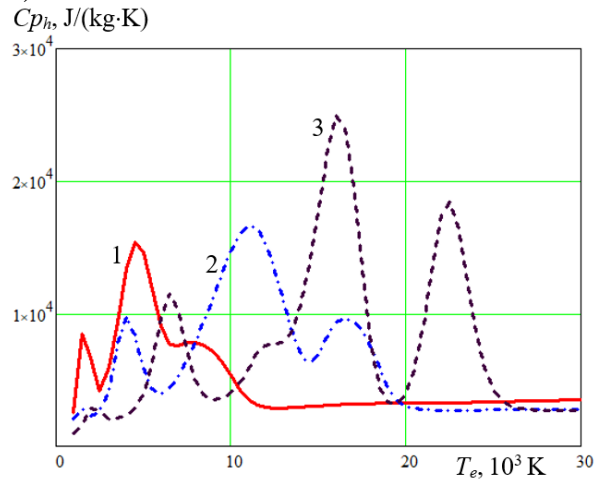

b)

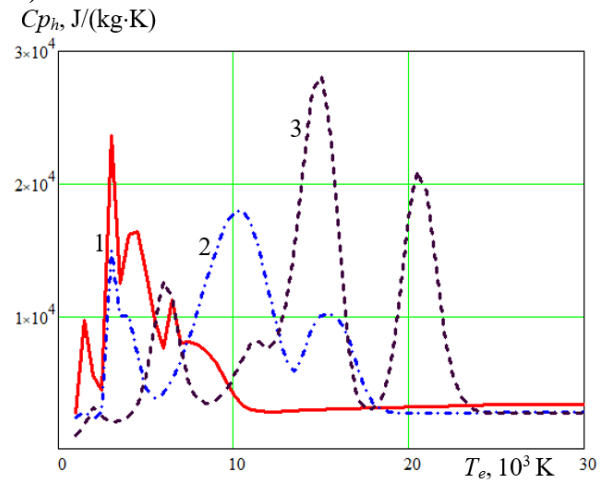

Fig. 5. Heavy particles specific heat dependence on electron temperature at different values of parameter $\theta(1-\theta=1 ; 2-\theta=3 ; 3-\theta=5)$ for different plasma pressures: $\mathrm{a}-1 \mathrm{~atm}, \mathrm{~b}-0.3 \mathrm{~atm}$

a)

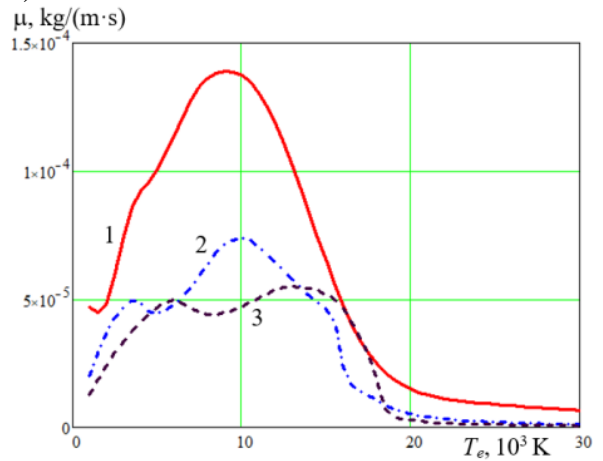

b)

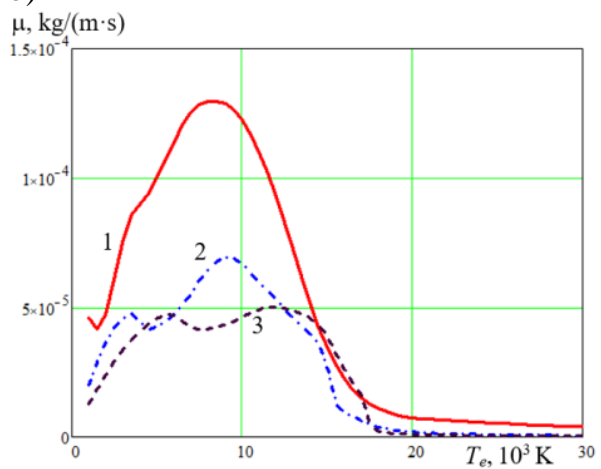

Fig. 6. Viscosity dependence on electron temperature at different values of parameter $\theta(1-\theta=1$; $2-\theta=3 ; 3-\theta=5$ ) for different plasma pressures: $\mathrm{a}-1 \mathrm{~atm}, \mathrm{~b}-0.3 \mathrm{~atm}$ 
a)

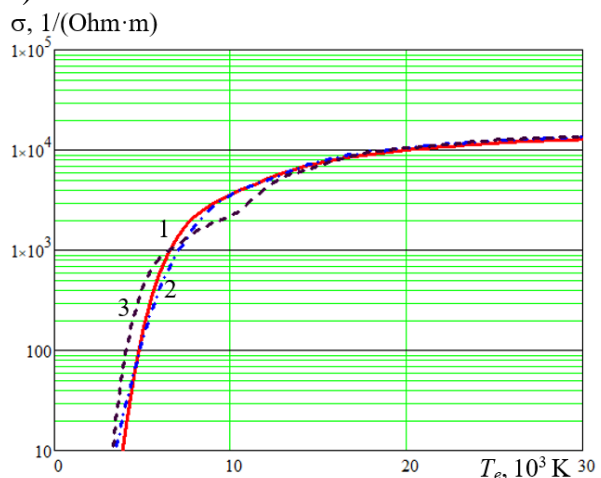

b)

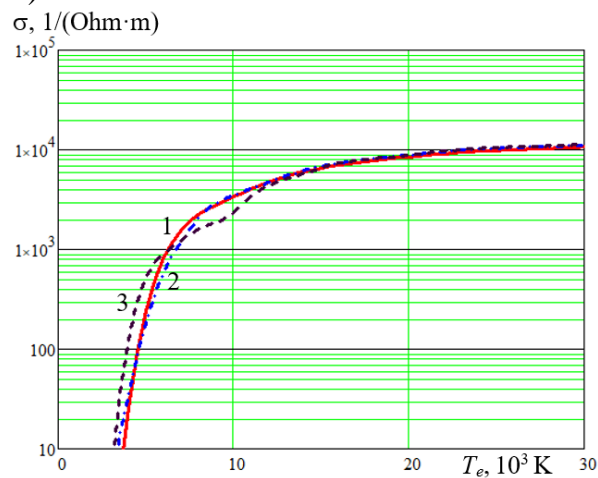

Fig. 7. Electrical conductivity dependence on electron temperature at different values of parameter $\theta$ $(1-\theta=1 ; 2-\theta=3 ; 3-\theta=5)$ for different plasma pressures: $a-1 \mathrm{~atm}, \mathrm{~b}-0.3 \mathrm{~atm}$

a)

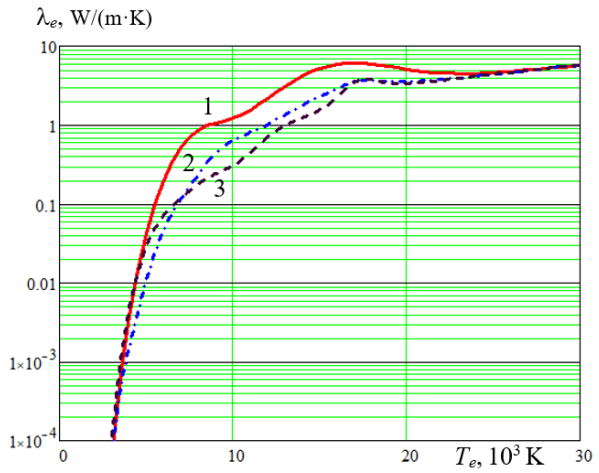

b)

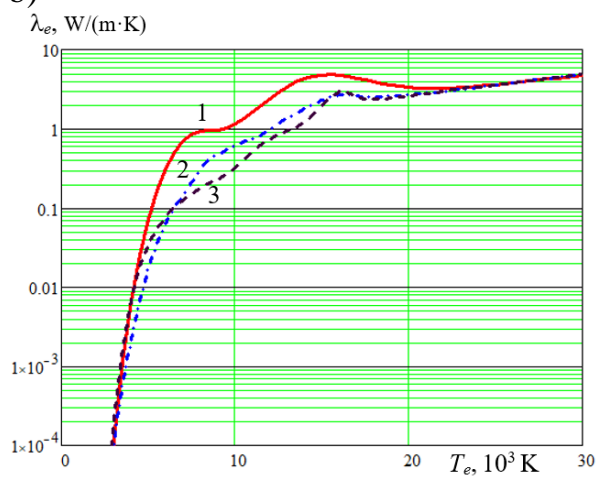

Fig. 8. Electron heat conductivity on electron temperature at different values of parameter $\theta$ $(1-\theta=1 ; 2-\theta=3 ; 3-\theta=5)$ for different plasma pressures: $a-1 \mathrm{~atm}, \mathrm{~b}-0.3 \mathrm{~atm}$

a)

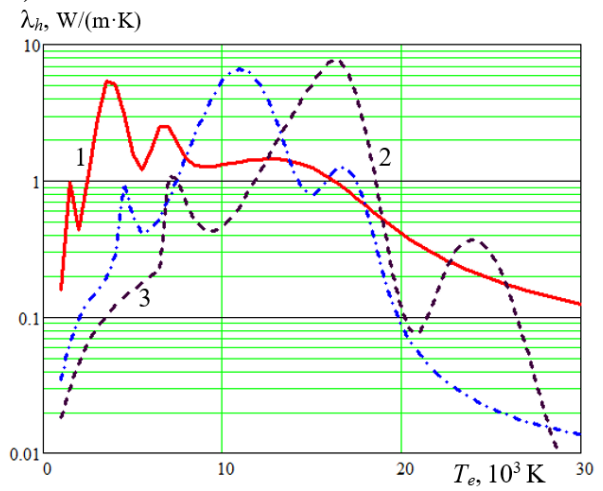

b)

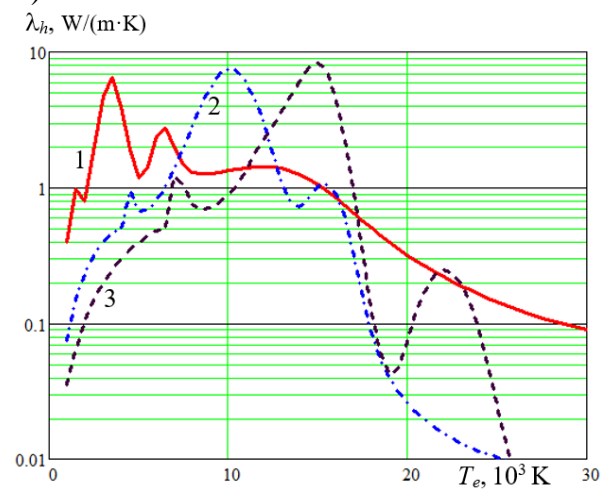

Fig. 9. Heavy particles heat conductivity dependence on electron temperature at different values of parameter $\theta(1-\theta=1 ; 2-\theta=3 ; 3-\theta=5)$ for different plasma pressures: $a-1 \mathrm{~atm}, \mathrm{~b}-0.3 \mathrm{~atm}$ 


\section{Conclusions}

The dependences of the thermodynamic and transport properties of plasma in a multichamber arrester were obtained for the two-temperature plasma model for the electron temperature range $T_{e}=300-30000 \mathrm{~K}$, the nonequilibrium degree $\theta=1-5$, and the pressure range $p=0.3-1$ atm.

The obtained dependences will be used to develop a model of a non-stationary twotemperature plasma in a discharge chamber of a multi-chamber arrester.

\section{References}

1. G.V. Podporkin, E.Yu. Enkin, E.S. Kalakutsky, V.E. Pilshikov, A.D. Sivaev, X Int. Symp. on Lightning Protection, 569-574 (2010).

2. R. Kozakov, A. Khakpour, S. Gorchakov, D. Uhrlandt, D. Ivanov, I. Murashov, G. Podporkin, V. Frolov, Plasma Phys. Technology, 2(2), 150-154 (2015)

3. G.V. Podporkin, Plasma Phys. Technology, 2(3), 241-250 (2015)

4. M.E. Pinchuk, A.V. Budin, I.I. Kumkova, (...), A.N. Chusov, R.I. Zaynalov, J. Phys.: Conf. Series 774(1), 012187 (2016)

5. V.Y. Frolov, D.V. Ivanov, Y.V. Murashov, A.D. Sivaev, Techn. Phys. Letters, 41(4), 310-313 (2015)

6. V. Frolov, D. Ivanov, I. Murashov, A. Sivaev, Proc. 2016 IEEE North West Russia Section Young Researchers in Electrical and Electronic Engineering Conf., ElConRusNW 2016, 562-565 (2016)

7. A.N. Chusov, G.V. Podporkin, M.E. Pinchuk, D.V. Ivanov, I. Murashov, V.Ya. Frolov, Proc. 33rd Int. Conf. on Lightning Protection (2016)

8. Zhiwei Guo, Xinping Long, Zhongdong Qian, Ning Qiu, AIP Advances, 6, 045117 (2016)

9. V.Y. Frolov, D.V. Ivanov, R.A. Belsky, Proc. 2017 IEEE Russia Section Young Researchers in Electrical and Electronic Engineering Conf., ElConRus 2017, 1520-1523 (2017)

10. N. Silin, N. Korovkin, T. Minevich, Proc. 2017 Int. Conf. on Industrial Engineering, Applications and Manufacturing, ICIEAM 2017, 8076412 (2017)

11. M.I. Boulos, P. Fauchais, E. Pfender, Thermal Plasmas: Fundamentals and Applications, Vol. 1. (1994)

12. E.V. Stupochenko, I.P. Stakhanov et al., Physical gas dynamics, 3-38 (1959) [in Russian].

13. Thermodynamic and Thermophysical Properties of Combustion Products, Ed. by V. P. Glushko, Vols. 1 (1971) [in Russian].

14. A.L. Suris, Thermodynamics of High-Temperature Processes (1985) [in Russian].

15. W.B. White, S.M. Johnson, G.B., J. Chem. Phys. 28(5), 751-755 (1958)

16. B. Pateyron, M.-F. Elchinger, G. Delluc, F. Fauchais, Plasma Chem. Plasma Process. 12(4), 421-448 (1992)

17. A.B. Murphy, C.J. Arundell, Plasma Chem. Plasma Process. 14(4), 451-490 (1994)

18. NIST Atomic Spectra Database: http://physics.nist.gov/PhysRefData/ASD/index.html

19. Thermodynamic Properties of Individual Substances: Reference Book, Ed. by V. P. Glushko et al., 3rd ed. (1978-1982), Vols. 1-4 [in Russian].

20. M.W. Chase, Jr., Editor. NIST-JANAF Thermochemical Tables, J. Phys. Chem. Ref. Data. Monograph No. 9 (Part I and II) (1998)

21. M. Capitelli, G. Colonna, A. D'Angola, Fundamental Aspects of Plasma Chemical Physics: Thermodynamics (2011).

22. S. Chapman, T.G. Cowling, The mathematical theory of non-uniform gases (1970) 
23. J.O. Hirschfelder, C.F. Curtiss, R.B. Bird, Molecular theory of gases and Liquids (1966)

24. R.S. Devoto, Phys. Fluids. 9(6), 1230-1240 (1966)

25. V. Rat, A.B. Murphy, J. Aubreton, M.F. Elchinger, P. Faushais, J. Phys. D: Appl. Phys. 41(18), 183001 (2008)

26. R.S. Devoto, Phys. Fluids. 10(10), 2105-2112 (1967)

27. J. Aubreton, M.F. Elchinger, P. Fauchais, Plasma Chem. Plasma Process. 18(1), 1-27 (1998)

28. R.S. Devoto, Phys. Fluids. 9(6), 1230-1240 (1966)

29. M. Capitelli, D. Bruno, A. Laricchiuta, Fundamental Aspects of Plasma Chemical Physics: Transport (2013)

30. Y. Cressault, R. Hannachi, Ph. Teulet, A. Gleizes, J.-P. Gonnet, J.-Y. Battandier, Plasma Sources Sci. Technol. 17, 035016 (2008)

31. P. Andre, J. Aubreton, S. Clain, M. Dudeck, E. Duffour, M.F. Elchinger, B. Izrar, D. Rochette, R. Touzani, D. Vacher, Eur. Phys. J. D, 57, 227-234 (2010)

32. E.A. Mason, R.J. Munn, F.J. Smith, Phys. Fluids. 10(8), 1827-1832 (1967) 